\title{
PARASITISME DES LORANTHACEAE DANS LES PLANTATIONS DE LEGUMINEUSES ARBORESCENTES EN ZONE FORESTIERE DE LA CÔTE D'IVOIRE
}

\author{
K. SORO', G. M. GNAHOUA² et D. TRAORE ${ }^{1}$ \\ ${ }^{1}$ Laboratoire de Botanique, U.F.R. Biosciences, Université de Cocody. 22 BP 582 Abidjan 22, Côte d'Ivoire. \\ E-mail : skafana1@yahoo.fr \\ ${ }^{2}$ Centre National de Recherche Agronomique, Station de Gagnoa. BP 602 Gagnoa, Côte d'Ivoire.
}

\begin{abstract}
RESUME
Les Légumineuses arborescentes produisent des fruits comestibles, du bois, du fourrage et fournissent l'essentiel du flux d'azote dans les écosystèmes. Elles sont utilisées dans les systèmes agroforestiers pour l'ombrage et la restauration de la fertilité. Cependant, ces Légumineuses arborescentes sont parasitées par les Loranthaceae, qui détruisent le feuillage, déprécient la qualité du bois et provoquent la mort de leurs hôtes. La présente étude, réalisée sur des Légumineuses arborescentes, plantées dans la zone forestière de la Côte d'Ivoire, a permis d'évaluer le taux et l'intensité d'infestation des Loranthaceae sur la station de Oumé, Centre National de Recherche Agronomique (CNRA). Les résultats montrent une forte sensibilité, aux Loranthaceae, des Légumineuses arborescentes exotiques (Acacia mangium, Acacia auriculaeformis, Albizia guachapele et Albizia lebbeck), tandis que les espèces locales (Albizia adianthifolia et Albizia zygia) ont été moins infestées. Les taux d'infestation des parcelles ont semblé être liés à l'emplacement en bordure d'exploitation, au degré d'enherbement et à la proximité des espaces agricoles fréquentés par les disséminateurs des Loranthaceae (oiseaux frugivores). La Légumineuse arborescente la plus infestée a été Albizia lebbeck et la Loranthaceae la plus infestante Tapinanthus bangwensis.
\end{abstract}

Mots clés : Légumineuses arborescentes, Loranthaceae, infestation, Côte d'Ivoire.

\section{ABSTRACT}

PARASITISM OF MISTLETOES IN LEGUMINOUS TREE PLANTATIONS IN THE FOREST ZONE OF CÔTE D'IVOIRE

Leguminous trees provide edible fruits, wood, forage and the main nitrogen in ecosystems. They are increasingly used in agroforestry systems as shade for trees and for soil reclamation. However, leguminous trees are often hosts of Mistletoes, vascular parasites that destroy foliages, depreciate wood quality and cause the death of their hosts. In this study on Leguminous trees, in a forest area of Côte d'Ivoire, infestation rates and intensities of Mistletoes were assessed at the Centre National de Recherche Agronomique (CNRA) station in the district of Oumé. Results show a high sensitivity of exotic species (Acacia mangium, Acacia auriculaeformis, Albizia guachapele and Albizia lebbeck) to Mistletoes, while native species (Albizia adianthifolia and Albizia zygia) were less infested. Infestation rates seemed to be related to the position at the exploitation border, the weediness of plots and the proximity of crop land that attract Mistletoes scattering birds. The most infested leguminous tree is Albizia lebbeck and the plentiful parasitic was Tapinanthus bangwensis.

Key words : Legumimous trees, Mistletoes, infestation, Côte d'Ivoire. 


\section{INTRODUCTION}

La forte dégradation de la forêt ivoirienne a de multiples conséquences sur le milieu écologique et les systèmes agraires : croissance anormale de la température ambiante, déficits hydriques prolongés et irrégularité des saisons pluvieuses (Lecomte, 1990). Cette situation a accru la mortalité dans les vergers de cultures d'exportation et marginalisé des régions autrefois grandes productrices de café et de cacao (Gnahoua, 1998).

Des Légumineuses arborescentes, à croissance rapide ont été introduites dans la zone forestière d'Oumé en vue d'accélérer la formation de jachères et de reconstituer le microclimat nécessaire à la replantation des cultures de rente. Cependant, ces Légumineuses arborescentes, notamment les Mimosaceae sont des hôtes privilégiés des Loranthaceae (Dembélé et al., 1994). Des espèces introduites à Oumé ont fait l'objet d'attaques de la part de ces parasites végétaux (Soro, 2005).

De nombreuses essences ligneuses, dont celles qui ont une importance économique ou entretenues par l'homme sont infestées par les Loranthaceae. Le degré d'infestation varie selon la nature de l'hôte, la dureté, l'âge et la hauteur du bois. Le parasitisme des Loranthaceae affecte la croissance de l'hôte qui peut mourir ou perdre sa valeur économique (Frochot et al., 1978).

La lutte efficace à mener contre ces parasites végétaux nécessite, de connaître la manifestation de leur infestation sur les différents hôtes. La présente étude vise à évaluer le taux et l'intensité d'infestation des Loranthaceae sur les Légumineuses arborescentes plantées dans la zone forestière d'Oumé.

\section{MATERIEL ET METHODES}

\section{SITE D'ETUDE}

La présente étude a été réalisée sur la station de recherche forestière du Centre National de Recherche Agronomique (CNRA) à Oumé. La station est située à $12 \mathrm{~km}$ de la ville de Oumé, sur l'axe routier Oumé-Gagnoa, dans le CentreOuest de la Côte d'Ivoire. Elle couvre 400 ha à l'intérieur de la forêt classée de la Sangoué, avec pour coordonnées géographiques : $06^{\circ} 17^{\prime}$ de latitude Nord et $05^{\circ} 31^{\prime}$ de longitude Ouest. Le climat est sub-équatorial à deux saisons de pluies (d'avril à juin et de septembre à octobre) qui alternent avec deux saisons sèches (de décembre à février et de juillet à août). La pluviométrie moyenne annuelle sur 33 ans (19702003) est de $1302,1 \mathrm{~mm}$. La température moyenne est de $27{ }^{\circ} \mathrm{C}$ (Gagnoa) et varie annuellement entre 19 et $33^{\circ} \mathrm{C}$ (Dabbadie, 1996).

\section{MATERIEL VEGETAL}

Le matériel végétal est constitué de 6 espèces de Légumineuses arborescentes appartenant à la famille des Mimosaceae dont 4 espèces exotiques et 2 locales. Les espèces exotiques concernées sont : Acacia auriculaeformis A. Cunn. ex Benth., Acacia mangium Willd., Albizia guachapele (Kunth) Dugand et Albizia lebbeck (L.) Benth. Les espèces locales sont : Albizia adianthifolia (Schumach.) W.F. Wright et Albizia zygia (DC.) J.F. Macbr.

\section{METHODES D'ETUDE}

La méthodologie de travail a consisté à dénombrer et à analyser les niveaux d'infestation des Loranthaceae parasites présentes sur les Légumineuses arborescentes de quatre dispositifs agroforestiers (Tableau 1 ). Après les comptages effectués dans l'ensemble des essais, les mesures et les observations ont été réalisées dans l'essai de régénération de la cacaoyère (de 11 ans) par des techniques agroforestières. Cet essai s'étend sur une superficie de 6 ha et comporte 1091 arbres et 6 espèces de Légumineuses arborescentes (Tableau 1).

\section{DISPOSITIF EXPERIMENTAL DE L'ESSAI LÉGUMINEUSES/CACOYERS}

Le dispositif expérimental de l'essai de régénération de la cacaoyère par des techniques agroforestières, est un factoriel. Deux facteurs ont été étudiés dans 4 blocs :

- le mode d'implantation des Légumineuses et - la Légumineuse arborescente.

Le premier facteur, constitué par le mode d'implantation des Légumineuses, comprend 4 modalités ou traitements (dispositifs agroforestiers) :

$\mathrm{T}_{1}$ : Complantation de Légumineuses arborescentes et de cacaoyers de 11 ans ; 
$\mathrm{T}_{2}$ : Complantation de Légumineuses arborescentes, de bananiers et de cacaoyers de 11 ans ;

$\mathrm{T}_{3}$ : Complantation de Légumineuses arborescentes et bananiers de 11 ans, puis introduction des cacaoyers après un différé d'un an (10 ans) ;

$\mathrm{T}_{4}$ : Haie vive de Légumineuses arborescentes autour des cacaoyers et bananiers, âgés de 11 ans.

La Légumineuse arborescente constitue le deuxième facteur, avec 6 modalités, qui correspondent au matériel végétal utilisé : Acacia auriculaeformis, Acacia mangium, Albizia guachapele, Albizia lebbeck, Albizia adianthifolia et Albizia zygia.

L'essai comporte 4 blocs. Les parcelles unitaires sont de $21 \mathrm{~m} \times 20 \mathrm{~m}\left(420 \mathrm{~m}^{2}\right)$. Le bloc I est implanté à l'intérieur de la station. Les blocs II, III et IV sont contigus et installés à la périphérie de la station, en regard des parcelles agricoles des villages riverains. Les individus des 6 espèces étudiées ont été plantés à la même densité et soumis aux mêmes traitements sylvicoles (éclaircies, élagages) visant à réduire l'ombrage au dessus des jeunes cacaoyers associés. Les Légumineuses arborescentes qui produisaient plus d'ombrage ont fait l'objet de coupes plus nombreuses et les densités résiduelles d'arbres diffèrent d'une espèce à l'autre. Certaines Légumineuses arborescentes abattues ont produit des rejets de souches et d'autres ont donné de nombreuses repousses naturelles à partir des graines. L'essai comporte donc des arbres issus de la plantation (arbres non régénérés), de rejets de souches et de semis naturels (arbres régénérés).

\section{VARIABLE MESUREE}

L'infestation par les Loranthaceae a été caractérisée et elle constitue la variable quantitative mesurée. Les comptages ont porté sur le nombre d'arbres infestés de chaque espèce qui est représenté par (i) et le nombre de touffes de parasites (vivants et morts) présents sur chaque arbre qui est représenté par (ii). Ces données ont permis de déterminer :

- le taux d'infestation qui est le pourcentage de plants infestés dans un essai ou au sein d'une espèce donnée ;
- l'intensité d'infestation qui exprime l'ampleur de l'infestation sur les individus parasités dans un bloc ou au sein d'une espèce. Elle est exprimée, ici, par le nombre moyen de touffes de Loranthaceae observées sur un individu ;

- le taux de mortalité naturelle des parasites, qui résulte du rapport nombre de touffes mortes sur le nombre total de touffes des parasites.

Les données mesurées par espèce et par traitement ont fait l'objet d'une analyse de variance à l'aide du logiciel XLSTAT version 7.5. Ce logiciel prévoit, en cas de différences significatives, une comparaison de moyennes par le test de Duncan au seuil $\alpha=5 \%$.

\section{RESULTATS}

Les observations faites sur les Légumineuses arborescentes de la station d'Oumé montrent que certaines d'entre elles sont infestées à divers degrés par 3 espèces de Loranthaceae à savoir : Tapinanthus bangwensis (Engl. \& K. Krause) Danser, Phragmanthera capitata var. alba (Spreng.) Ballé et Globimetula dinklagei subsp. assiana (Engl.) Danser.

\section{TAUXD'INFESTATION DES LORANTHACEAE DANS LES DISPOSITFS AGROFORESTIERS}

L'état du parasitisme des Loranthaceae dans les différents dispositifs agroforestiers de la station montre que le taux d'infestation de $63,20 \%$ est observé dans l'essai Légumineuses/caféiers (Tableau 1). L'essai Légumineuses/cacaoyers a enregistré un taux de $29,05 \%$. Celui trouvé dans l'essai Légumineuses arborées s'élève à $11,11 \%$. Le taux de $4,87 \%$ est obtenu dans l'essai jachères arborées. Le taux moyen d'infestation des Légumineuses arborescentes dans les essais prospectés est de $27,17 \%$. Toutefois, l'essai Légumineuses/cacaoyers comporte 317 individus infestés sur un total de 393 pieds parasités pour l'ensemble des essais (Tableau 1). Quatre espèces exotiques (Acacia mangium, Acacia auriculaeformis, Albizia guachapele et Albizia lebbeck) sont infestées par les Loranthaceae. Les 2 espèces locales (Albizia adianthifolia et Albizia zygia) sont exemptes des Loranthaceae (Tableau 1). 
Tableau 1 : Caractéristiques, nombre d'individus parasités et taux d'infestation par les Loranthaceae des Légumineuses arborescentes dans les différents dispositifs agroforestiers en zone de transition.

Characteristics, parasitized individuals and infestation rate of Leguminous trees in Agroforestry trials.

\begin{tabular}{|c|c|c|c|c|c|c|c|c|c|c|c|c|}
\hline \multirow[b]{2}{*}{ Intitulés des essais } & \multirow{2}{*}{$\begin{array}{c}\text { Âges des } \\
\text { essais }\end{array}$} & \multirow{2}{*}{$\begin{array}{l}\text { Superficie } \\
\text { (ha) }\end{array}$} & \multirow{2}{*}{$\begin{array}{l}\text { Nombre } \\
\text { d'espèces } \\
\text { présentes }\end{array}$} & \multirow{2}{*}{$\begin{array}{c}\text { Arbres } \\
\text { présents }\end{array}$} & \multicolumn{6}{|c|}{ Individus parasités } & \multirow{2}{*}{$\begin{array}{c}\text { Total } \\
\text { d'arbres } \\
\text { infestés }\end{array}$} & \multirow{2}{*}{$\begin{array}{c}\text { Taux } \\
\text { d'infestation } \\
(\%)\end{array}$} \\
\hline & & & & & $\begin{array}{l}\text { Ac. } \\
\text { au. }\end{array}$ & $\begin{array}{l}\text { Ac. } \\
m a .\end{array}$ & $\begin{array}{l}A l . \\
g u .\end{array}$ & $\begin{array}{l}A l . \\
l e .\end{array}$ & $\begin{array}{l}\text { Al. } \\
\text { ad. }\end{array}$ & $\begin{array}{l}\text { Al. } \\
z y .\end{array}$ & & \\
\hline Légumineuses / cacaoyers & 11 & 6 & 6 & 1091 & 72 & 50 & 87 & 108 & 0 & 0 & 317 & 29,05 \\
\hline Légumineuses / caféiers & 8 & 2,5 & 4 & 106 & - & - & 32 & 35 & 0 & 0 & 67 & 63,21 \\
\hline Jachères arborées & 14 & 5 & 2 & 123 & 1 & 5 & - & - & - & - & 6 & 4,88 \\
\hline Légumineuses arborées & 17 & 1,5 & 2 & 27 & 1 & 2 & - & - & - & - & 3 & 11,11 \\
\hline Total & & & & 1347 & 74 & 57 & 119 & 143 & 0 & 0 & 393 & 29,17 \\
\hline Moyenne & & & & 336,7 & 24,7 & 19,0 & 59,5 & 71,5 & 0 & 0 & 98,2 & 29,17 \\
\hline
\end{tabular}

Ac. au. : Acacia auriculaeformis ; Ac. ma. : Acacia mangium; Al. gu. : Albizia guachapele ; Al. le. : Albizia lebbeck ; Al. ad. : Albizia adianthifolia; Al. zy. : Albizia zygia. - : Absence d'individu parasité dans l'essai considéré

\section{ETAT DU PARASITISME DES LORANTHACEAE DANS L'ESSAI LEGUMINEUSES/CACAOYERS}

La distribution des Loranthaceae dans l'essai Légumineuses / cacaoyers (Tableau 2) montre que les 3 espèces parasites sont présentes sur les 4 Légumineuses exotiques. Celles-ci totalisent 3411 touffes vivantes dont 3242 soit 95,04\% pour Tapinanthus bangwensis. Les touffes mortes sont au nombre de 1541 soit $31,12 \%$ du nombre total de touffes. Les touffes desséchées ne permettent pas d'identifier le parasite concerné. Elles sont totalisées par espèce de Légumineuses arborescentes pour l'estimation ultérieure du taux de mortalité. Ces touffes sont au nombre de 598 touffes soit $39,50 \%$ des touffes mortes chez Acacia auriculaeformis et 277 touffes soit $18,29 \%$ sur Albizia lebbeck.

\section{TAUXD'INFESTATION}

Les données (Tableau 3) indiquent qu'il y a un effet «taux d'infestation» sur les traitements. Trois groupes statistiques ont été constitués. Les traitements 2 et 3 forment le premier groupe. Les traitements 4 et 1 constituent respectivement le deuxième et le troisième groupe.

II y a également un effet «taux d'infestation» sur les espèces de Légumineuses arborescentes (Tableau 3). Trois groupes statistiques sont identifiés. En effet, les taux d'infestation de
Acacia auriculaeformis, Albizia lebbeck et Acacia mangium constituent le premier groupe statistique. Le deuxième groupe comporte Albizia guachapele (Tableau 3). Le troisième groupe rassemble Albizia adianthifolia et Albizia zygia dont les taux d'infestation sont nuls.

Dans le dispositif Légumineuses arborescentes/ cacaoyers, les arbres non régénérés ont présenté un taux d'infestation de 73,24\%. Les taux d'infestation vont de $68 \%$ chez Acacia mangium à $83 \%$ chez Acacia auriculaeformis. Les arbres régénérés ont enregistré un taux de $30,66 \%$. Les taux d'infestation des arbres régénérés oscillent autour de $6 \%$ pour Albizia guachapele et $44 \%$ pour Acacia mangium (Figure 1).

\section{TAUX DE MORTALITE NATURELLE DES LORANTHACEAE}

Les résultats de l'analyse de variance effectuée sur les taux de mortalité des Loranthaceae (Tableau 3) montrent qu'il y a un effet «taux de mortalité» sur les espèces de Légumineuses arborescentes. Quatre groupes statistiques sont constitués. Acacia mangium et Albizia lebbeck représentent respectivement le premier et le deuxième groupe. Acacia auriculaeformis et Albizia guachapele sont statistiquement identiques et composent le troisième groupe statistique. Les 2 espèces locales ne portent aucune touffe morte de Loranthaceae et constituent le quatrième groupe. 
Tableau 2 : Etat du parasitisme des Loranthaceae sur les espèces de Légumineuses arborescentes dans l'essai Légumineuses / cacaoyers.

Parasitism inventory of on legume trees species in the Leguminous / cocoa association test.

\begin{tabular}{|c|c|c|c|c|c|c|c|}
\hline \multirow{2}{*}{$\begin{array}{l}\text { Espèces de } \\
\text { Légumineuses } \\
\text { arborescentes }\end{array}$} & \multirow{2}{*}{$\begin{array}{c}\text { Arbres } \\
\text { présents }\end{array}$} & \multirow{2}{*}{$\begin{array}{l}\text { Arbres } \\
\text { infestés }\end{array}$} & \multicolumn{3}{|c|}{ Nombre de touffes vivantes de Loranthaceae } & \multirow{2}{*}{$\begin{array}{l}\text { Touffes } \\
\text { mortes }\end{array}$} & \multirow{2}{*}{$\begin{array}{l}\text { Total } \\
\text { touffes }\end{array}$} \\
\hline & & & $\begin{array}{c}\text { Tapinanthus } \\
\text { bangwensis }\end{array}$ & $\begin{array}{c}\text { Phragmanthera } \\
\text { capitata }\end{array}$ & $\begin{array}{c}\text { Globimetula } \\
\text { dinklagei }\end{array}$ & & \\
\hline Acacia auriculaeformis & 105 & 72 & 307 & 2 & 18 & 598 & 925 \\
\hline Acacia mangium & 81 & 50 & 720 & 4 & 16 & 277 & 1017 \\
\hline Albizia lebbeck & 168 & 108 & 1319 & 32 & 47 & 321 & 1719 \\
\hline Albizia guachapele & 322 & 87 & 896 & 4 & 46 & 318 & 1264 \\
\hline Albizia adianthifolia & 225 & - & - & - & - & - & - \\
\hline Albizia zygia & 190 & - & - & - & - & - & - \\
\hline Total & 1091 & 317 & 3242 & 42 & 127 & 1514 & 4925 \\
\hline Moyenne & 181,8 & 79,7 & 810,5 & 10,5 & 31,7 & 378,5 & 1231,2 \\
\hline
\end{tabular}

- : Absence de l'individu parasité dans l'essai considéré

Tableau 3 : Moyennes des taux d'infestation et de mortalité sur les 6 Légumineuses arborescentes étudiées dans les 4 associations culturales de l'essai Légumineuses / cacaoyers.

Mean infestation rate and mortality rate of Mistletoes on 6 studied Leguminous trees in 4 agricultural associations of the Leguminous / cocoa association test.

\begin{tabular}{|c|c|c|c|c|}
\hline Facteurs étudiés & Taux d'infestation & $\begin{array}{c}\text { Degrés de } \\
\text { signification }\end{array}$ & $\begin{array}{l}\text { Taux de } \\
\text { mortalité }\end{array}$ & $\begin{array}{l}\text { Degrés de } \\
\text { signification }\end{array}$ \\
\hline \multicolumn{5}{|l|}{$\begin{array}{l}\text { Facteur } 1 \text { : Associations } \\
\text { culturales }(\mathrm{AC})\end{array}$} \\
\hline $\mathrm{T} 2$ & 0,409 a & \multirow{4}{*}{$*$} & 0,202 & \multirow{4}{*}{ ns } \\
\hline $\mathrm{T} 3$ & $0,437 \mathrm{a}$ & & 0,200 & \\
\hline $\mathrm{T} 1$ & $0,364 \mathrm{ab}$ & & 0,207 & \\
\hline $\mathrm{T} 4$ & $0,284 \quad b$ & & 0,143 & \\
\hline \multicolumn{5}{|l|}{$\begin{array}{l}\text { Facteur } 2 \text { : Espèces de } \\
\text { Légumineuses (EL) }\end{array}$} \\
\hline Acacia auriculaeformis & $0,68 \mathrm{a}$ & \multirow{6}{*}{$* * *$} & $0,30 \mathrm{ab}$ & \multirow{6}{*}{$* * *$} \\
\hline Albizia lebbeck & $0,64 \mathrm{a}$ & & $0,15 \mathrm{~b}$ & \\
\hline Acacia mangium & $0,61 \mathrm{a}$ & & $0,42 \mathrm{a}$ & \\
\hline Albizia guachapele & $0,27 \mathrm{~b}$ & & $0,25 \mathrm{ab}$ & \\
\hline Albizia adianthifolia & $\mathrm{c}$ & & $\mathrm{c}$ & \\
\hline Albizia zygia & 0 & & $\mathrm{c}$ & \\
\hline $\mathrm{AC} \times \mathrm{EL}$ & ns & & ns & \\
\hline Moyenne générale & 0,37 & & 0,18 & \\
\hline C.V. $(\%)$ & 100,80 & & 124,46 & \\
\hline
\end{tabular}

Les moyennes affectées de la même lettre ne sont pas différentes par le test de Duncan, à $5 \%$; ns : Non Significatif ; ${ }^{*}$ Significatif ; *** : Très Hautement Significatif ; C.V. : Coefficient de Variation.

$T_{1}$ : Complantation de Légumineuses et de cacaoyers en 1993 ;

$\mathrm{T}_{2}$ : Complantation de Légumineuses, de bananiers et de cacaoyers en 1993 ;

$\mathrm{T}_{3}$ : Complantation de Légumineuses et bananiers en 1993, puis introduction des cacaoyers après un différé d'un an (1994) ;

$\mathrm{T}_{4}$ : Haie vive de Légumineuses autour des cacaoyers et bananiers, plantés en 1993. 


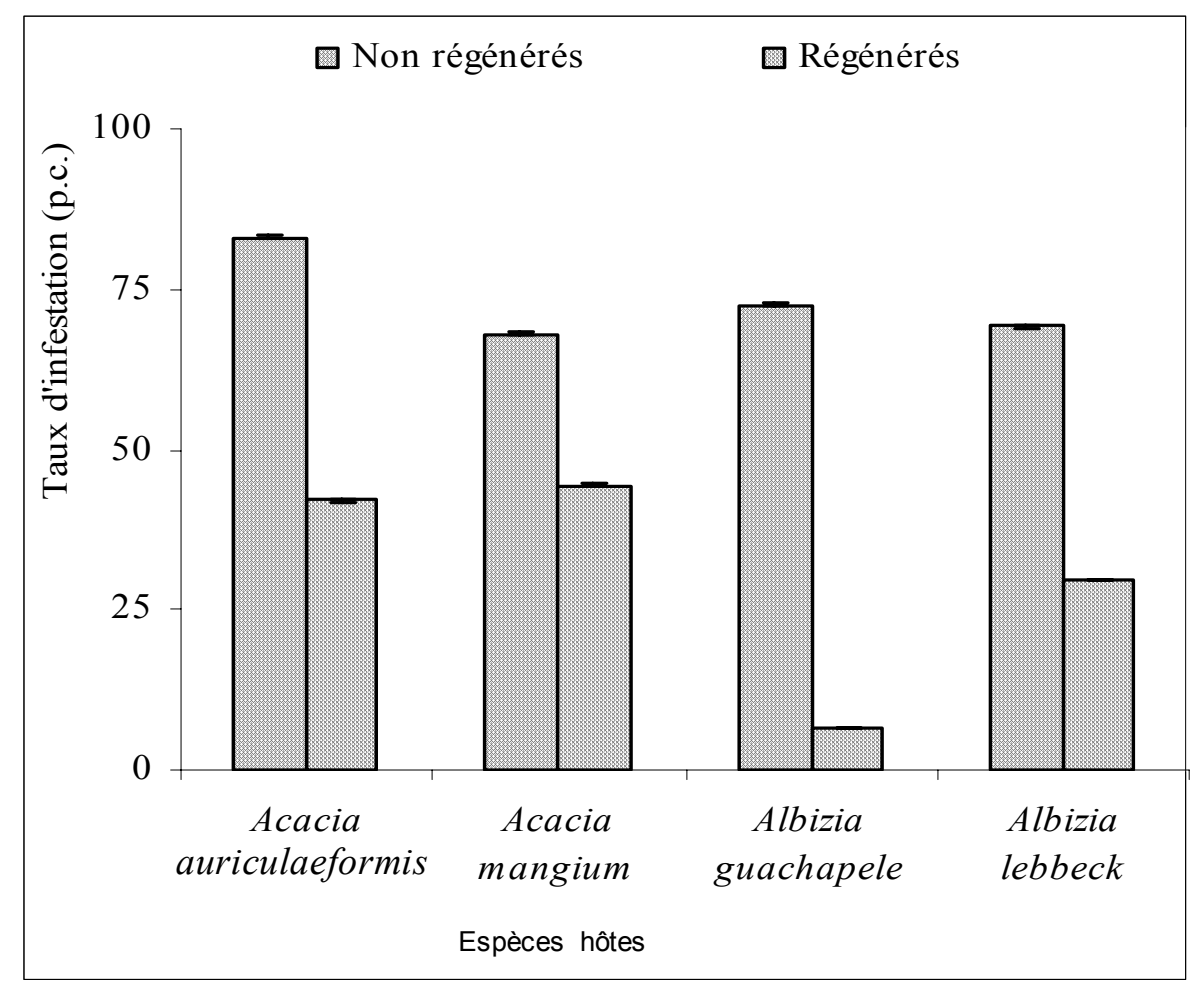

Figure 1 : Taux d'infestation des arbres non régénérés et des arbres régénérés dans l'essai Légumineuses / cacaoyers.

Infestation rate of non regenerated trees and regenerated trees in the Leguminous / cocoa association test.

\section{INTENSITE D'INFESTATION DES ESPECES DE LEGUMINEUSES ARBORESCENTES}

L'intensité d'infestation exprimée par le nombre de touffes de Loranthaceae (vivantes et mortes) par arbre, montre que les 3 parasites identifiés sont présents sur les 4 Légumineuses arborescentes infestées (Tableau 4). Cependant, Tapinanthus bangwensis porte 5,82 touffes en moyenne par arbre. Globimetula dinklagei enregistre 0,23 touffes et Phragmanthera capitata compte 0,16 touffes en moyenne par arbre.

L'intensité d'infestation ne discrimine pas les traitements. Cependant, il y a un effet «intensité d'infestation» sur les espèces de Légumineuses arborescentes.

Pour Tapinanthus bangwensis, 5 groupes statistiques sont constitués. Le premier groupe est représenté par Albizia lebbeck. Le deuxième groupe comporte Albizia guachapele. Le troisième groupe compte Acacia auriculaeformis. Le quatrième groupe comprend Acacia mangium et le cinquième groupe rassemble les deux espèces locales d'Albizia qui ne sont pas infestées.

Pour Phragmanthera capitata, 3 groupes statistiques sont formés. Albizia lebbeck constitue le premier groupe. Acacia auriculeaformis et Albizia guachapele représentent le deuxième groupe. Acacia mangium et les deux Albizia locaux qui ne sont pas infestés, constituent le troisième groupe statistique.

Pour Globimetula dinklagei, 5 groupes statistiques sont composés. Le premier groupe est composé de Albizia guachapele. Le deuxième groupe est représenté par Albizia lebbeck. Le troisième groupe est constitué par Acacia mangium. Le quatrième groupe est représenté par Acacia auriculaeformis. Le cinquième groupe se compose des deux espèces locales qui ne sont pas infestées. 
Concernant l'intensité moyenne d'infestation de tous les parasites, 4 groupes statistiques sont constitués. Albizia lebbeck et Acacia auriculaeformis constituent le premier groupe. Albizia guachapele représente le deuxième groupe. Acacia mangium compose le troisième groupe. Les deux Albizia locaux qui ne sont pas infestés par les Loranthaceae, représentent le quatrième groupe statistique.

L'observation de l'intensité d'infestation des espèces à l'échelle du dispositif Légumineuses/ cacaoyers (Figure 2) met en relief l'existence d'un gradient de contamination des parcelles (Figure 3).

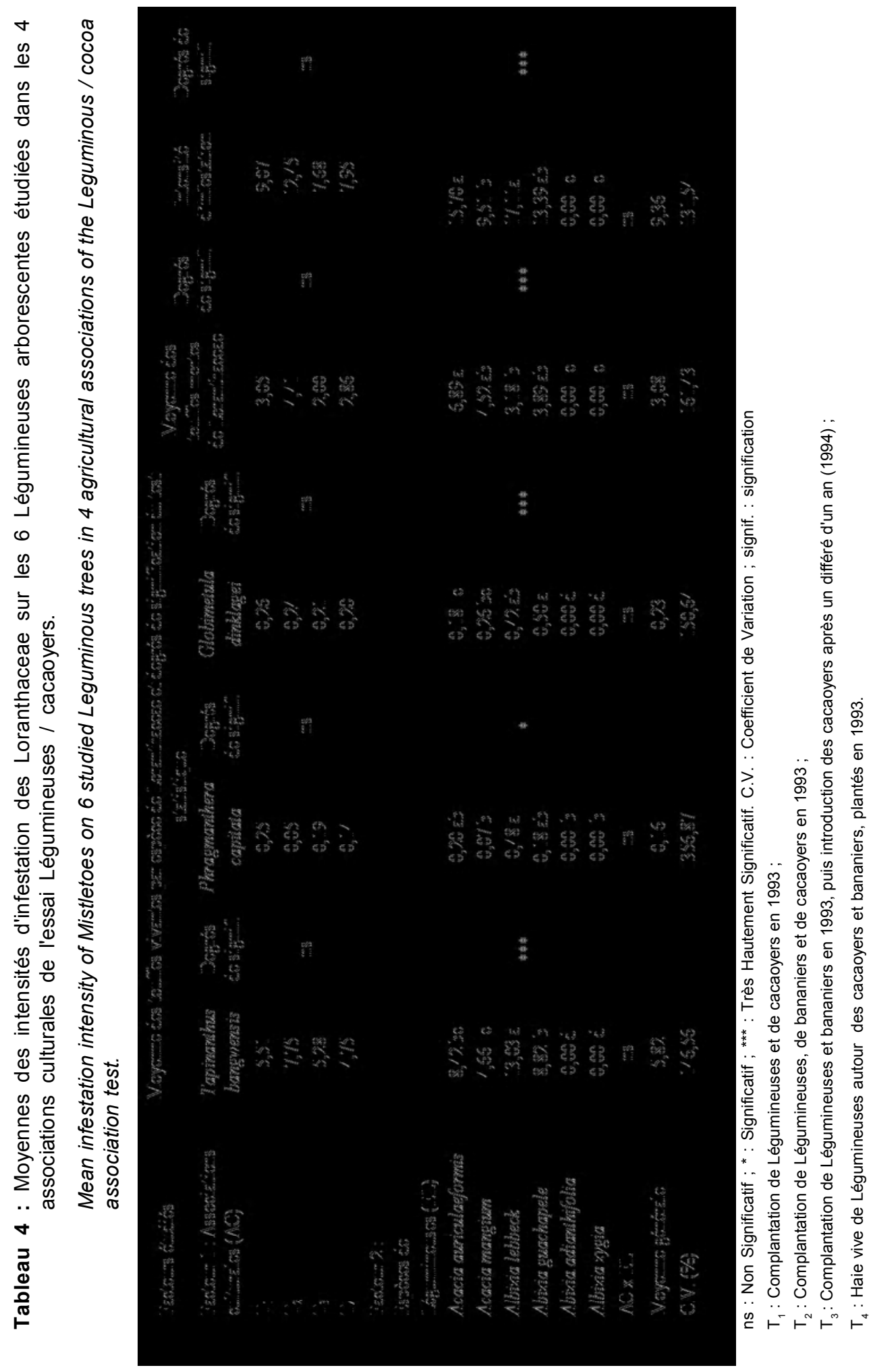




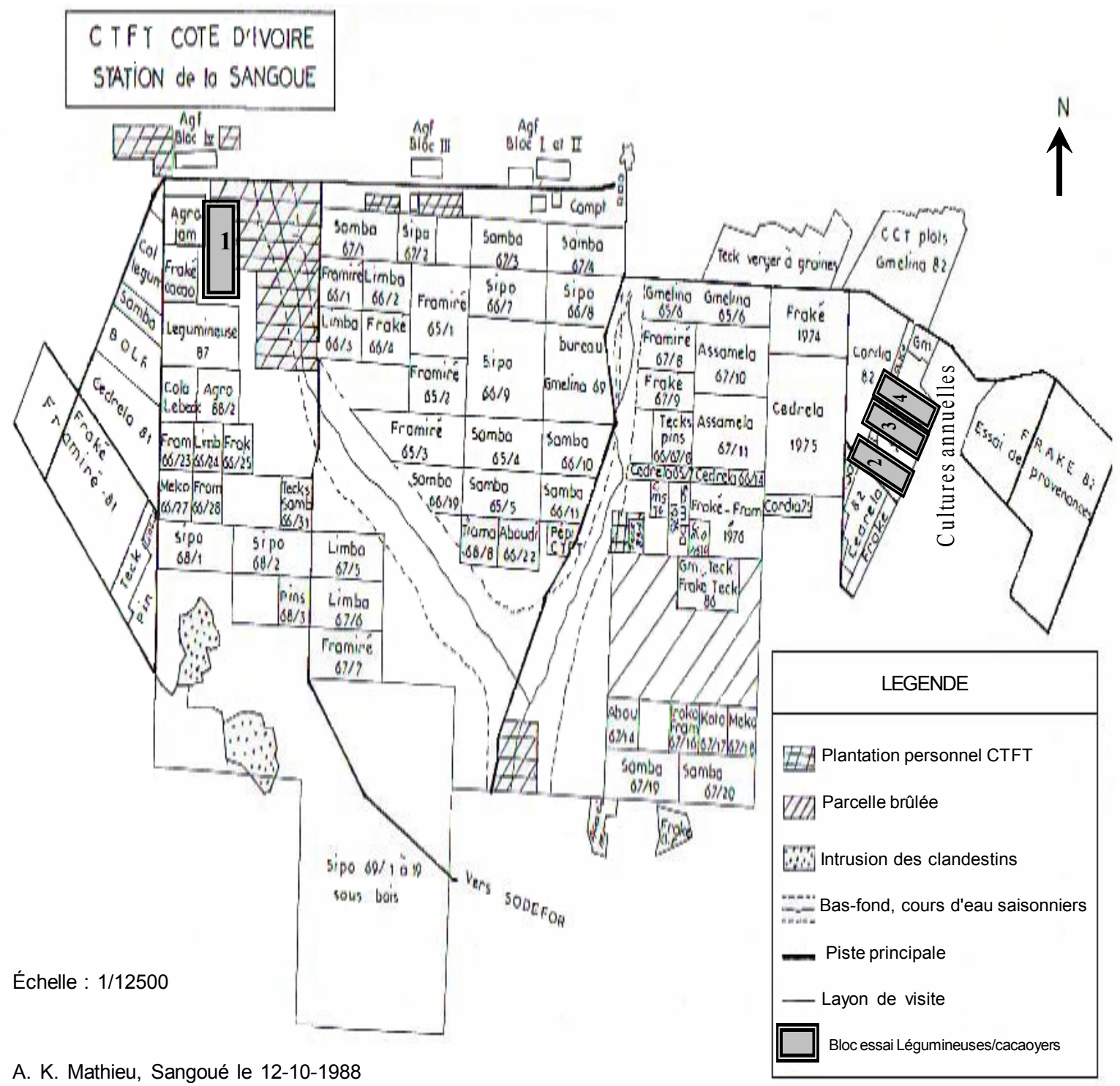

Modifié par S. Kafana le 28-11-2008

Figure 2 : Localisation des blocs de l'essai d'association arborée Légumineuses / cacaoyers dans la station annexe du CNRA à la Sangoué-Oumé.

Localization of Legumes / cocoa association tests in the CNRA station at Sangoué-Oumé. 


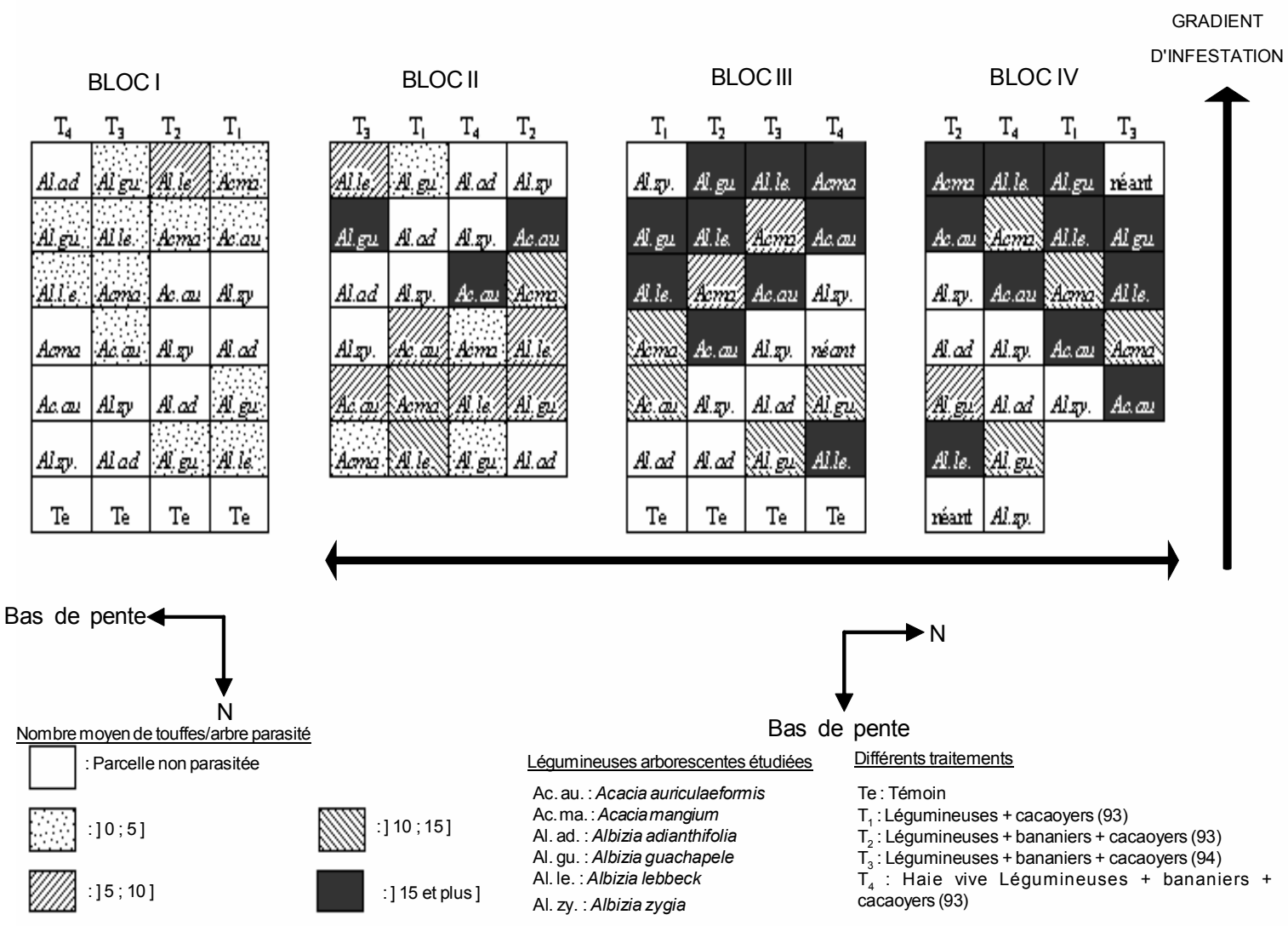

Figure 3 : Intensité d'infestation dans les différents traitements de l'essai d'association arborée Légumineuses / cacaoyers.

Infestation intensity in the plots of the Leguminous / cocoa association test.

\section{DISCUSSION}

De manière générale, cette étude a montré une forte sensibilité des Légumineuses arborescentes exotiques plantées en zone de transition forêt-savane à Oumé, aux Loranthaceae. Les différences observées dans la manifestation des attaques sur les Légumineuses arborescentes dénotent de la part de ces hôtes des différences de constitution des tissus. Ainsi, Albizia lebbeck qui est l'hôte le plus infesté, semble plus tolérant vis-à-vis de ces parasites tandis que les 2 Albizia locaux en sont plus résistants. La préférence des Loranthaceae pour Albizia lebbeck est confirmée par Houénon (1997) qui a signalé que cette espèce est l'essence forestière la plus menacée au Bénin par
Tapinanthus bangwensis, Tapinanthus globiferus et Agelanthus dodoneifolius.

Tapinanthus bangwensis est l'espèce la plus infestante parmi les 3 Loranthaceae recensées. Ce fort parasitisme de Tapinanthus bangwensis a été montré par Soro et al. (2004b) qui affirment que ce parasite constitue avec Agelanthus dodoneifolius les 2 parasites les plus rencontrés dans le parc naturel à Karité de Tengréla.

Le taux d'infestation global de ces Légumineuses arborescentes est 37,4 \%. Ce taux est supérieur au taux de 10,4 \% obtenu par Koffi (2004) sur l'Hévéa parasité par Tapinanthus bangwensis et Phragmanthera capitata, au Sud de la Côte d'Ivoire. Cependant, il est inférieur au taux d'infestation de $96 \%$ des 
7 parasites (Globimetula braunii, Globimetula cupulata, Phragmanthera capitata, Tapinanthus bangwensis, Tapinanthus dodoneifolius, Tapinanthus globiferus, Tapinanthus pentagonia) sur le karité et le néré, au Nord de la Côte d'Ivoire (Traoré et al., 2003).

Le taux moyen de mortalité naturelle des parasites s'est situé à $18,8 \%$ pour l'ensemble des Légumineuses arborescentes étudiées. La mortalité des parasites est plus élevée sur les Légumineuses arborescentes exotique en zone de transition $(28,8 \%)$ que sur l'Hévéa d'Anguédédou où Koffi (2004) a obtenu un taux moyen de $24 \%$. Ces taux de mortalité des Loranthaceae dénotent des comportements variables du parasite en fonction de l'espèce de Légumineuses arborescentes. Les taux de mortalité des parasites, élevés sur l'Acacia mangium et faibles sur l'Albizia lebbeck, montrent deux caractères opposés de ces 2 Légumineuses arborescentes. II s'agit du feuillage dense et du bois peu dur chez Acacia mangium et le feuillage clairsemé et le bois plus dur chez l'Albizia lebbeck. Les parasites ont aussi des exigences écologiques qui peuvent être réunies sur l'hôte et entraîner une mortalité faible ou importante. Selon Boussim (2002), la mortalité de Tapinanthus bangwensis s'explique, en partie, par le fait que les parties des branches situées en aval des points de fixation du parasite se dessèchent rapidement et exposent le parasite aux exigences hygrométriques très fortes et à un éclairement excessif.

Inversement, le Tapinanthus bangwensis a été plus rencontré à l'intérieur du houppier et Phragmanthera capitata a été plus observé à l'extérieur du houppier. Selon Koffi (2004), Phragmanthera capitata est une espèce héliophile qui se développe à la périphérie du houppier dans les plantations d'Hévéa tandis que Tapinanthus bangwensis est une espèce sciaphile. Globimetula dinklagei se rencontre à l'intérieur comme à l'extérieur du houppier. L'indifférence de position sur le houppier de ce parasite s'explique par le fait que ce dernier est une espèce de zone humide qui se rencontre jusqu'à 1000 km au dessus du littoral (Boussim, 2002).

L'intensité d'infestation des Loranthaceae observée sur les Légumineuses arborescentes en zone de transition est située à 9,36 touffes par arbre. Ce taux d'infestation est supérieur à celui obtenu par Koffi (2004) qui est de 1 touffe par pied d'Hévéa à Anguédédou au Sud de la
Côte d'Ivoire et inférieur au taux observé par Traoré et $\mathrm{Da}$ (2003) qui est de 24,2 touffes par pied de karité au Nord du pays. Les Légumineuses arborescentes étudiées sont donc moins atteintes que les sujets du parc à karité de Tingréla et plus parasitées que l'Hévéa de la station d'Anguédédou.

Pour les arbres non régénérés, on constate que les 4 espèces exotiques sont fortement parasitées. Ainsi, chez Albizia guachapele, qui est une espèce à forte régénération naturelle, les taux d'infestation relativement faibles peuvent être imputés à l'abondance des repousses de cette espèce. La forte infestation des arbres qui n'ont subi aucune coupe depuis leur mise en place peut être liée au fait qu'ils servent de perchoirs aux oiseaux à cause de leur hauteur plus importante. L'infestation plus importante des parcelles situées à la périphérie de la station (les blocs II, III et IV) serait le fait de la proximité des parcelles agricoles de riz et de maïs, qui attirent les oiseaux frugivores disséminateurs de Loranthaceae. En effet, selon Soro et al. (2004a), ces oiseaux ne s'aventurent pas à l'intérieur des peuplements forestiers fermés pour des raisons de sécurité et utilisent comme perchoirs, les grands arbres qui bordent les exploitations agricoles.

\section{CONCLUSION}

Cette étude montre que les Loranthaceae constituent une menace réelle pour les espèces exotiques de Légumineuses arborescentes étudiées (Acacia mangium, Acacia auriculaeformis, Albizia lebbeck et Albizia guachapele). Elle met également en relief le caractère résistant des 2 espèces de Légumineuses arborescentes locales (Albizia adianthifolia et Albizia zygia) aux 3 espèces de Loranthaceae recensées. Tapinanthus bangwensis est le parasite le plus infestant alors que les attaques de Phragmanthera capitata et Globimetula dinklagei sont très réduites en intensité. Parmi les Légumineuses arborescentes, Albizia lebbeck et Albizia guachapele sont les plus parasitées.

Les taux d'infestation des Loranthaceae, très élevés sur certaines Légumineuses arborescentes utilisées dans les techniques agroforestières constituent une menace pour les cultures associées (caféiers et cacaoyers) et 
pour les nouveaux itinéraires agricoles, l'arbre fixateur d'azote peut devenir un vecteur de Phanérogames parasites. Une des mesures de prévention des attaques dans les associations agroforestières est le recépage sélectif des grands arbres servant de perchoirs aux oiseaux disséminateurs de Loranthaceae.

En outre, le caractère résistant aux Loranthaceae de certaines Légumineuses arborescentes doit constituer un critère de choix de la composante ligneuse dans les associations agroforestières. Dans cette optique, la résistance aux Loranthaceae des Albizia locaux offrent une voie d'investigation vers de nouvelles espèces à promouvoir.

\section{REMERCIEMENTS}

Les auteurs remercient la direction du Centre National de Recherche Agronomique, le Professeur Aké-Assi L. et le personnel du site CNRA de la Sangoué-Oumé.

\section{REFERENCES}

Boussim I. J. 2002. Les Phanérogames parasites du Burkina Faso : inventaire, taxonomie, écologie, et quelques aspects de leur biologie : Cas particulier des Loranthaceae parasites du karité. Thèse de Doctorat d'État ès Sciences Naturelles, FA.S.T., Université de Ouagadougou, Burkina Faso, $285 \mathrm{p}$.

Dabbadie M. L. 1996. Étude de la viabilité d'une pisciculture rurale à faible niveau d'intrant dans le Centre-Ouest de la Côte d'Ivoire : Approche du réseau trophique. Thèse de Doctorat, option Océanologie biologique, Université Paris 6, $214 \mathrm{p}$.

Dembélé B., Raynal-Roques A., Salle G. et C. Tuquet. 1994. Plantes parasites des cultures et des semences forestières au Sahel. Institut du Sahel Bamako, Mali, C.T.A., Wageningen, The Netherlands, $43 \mathrm{p}$.

Frochot H., Pitch M. et L. Wehrlen. 1978. Différence de sensibilité au Viscum album L. de quelques clones de peupliers Populus sp. Bibliothèque nationale, Paris, Compte rendu du $103 e$ congrès national des Sociétés Savantes, Nancy-Metz, Tome 1 : pp. 7 - 165.

Gnahoua G. M. 1998. Agroforesterie en zone subhumide de Oumé In : Baumer M., Visite d'étude sur l'agroforesterie en Côte d'Ivoire. Compte rendu, Atelier du 16-27 septembre 1996, C.T.A./ A.C.P./U.E., pp 175 - 183.

Houénon G. J. 1997. Rapport d'une mission de recherche sur les parasites vasculaires de la famille des Loranthaceae dans la Sous-Préfecture de Bassila CPU/UNBBÉNIN, 5 p.

Koffi A. A. 2004. Évaluation de l'incidence des Loranthaceae sur la productivité de Hevea brasiliensis (Kunth) Müll. Arg. à Anguédédou (Sud de la Côte d'Ivoire). Mémoire de D.E.A. de Botanique, Université de CocodyAbidjan, $52 \mathrm{p}$.

Lecomte P. 1990. Place et intégration de l'arbre dans l'exploitation agricole ivoirienne du Centre-Ouest. Mém. C.N.E.A.R.C., Montpellier, France, 108 p.

Soro D., N'Da Adopo A, Da K. P. et D. Traoré. 2004a. Lutte contre les parasites chez le karité. Agronomie Africaine 16 (3) : 21 -28.

Soro D., Ouattara D., P. K. Da et D. Traoré. 2004b. Efficacité de l'émondage contre les Loranthaceae ou guis du karité : cas du parc naturel à karité de Tengrela dans le Nord de la Côte d'Ivoire. Annales de Botanique de l'Afrique de l'Ouest $3: 87$ - 95.

Soro K. 2005. Les Loranthaceae des Légumineuses arborescentes introduites dans la zone forestière d'Oumé : espèces rencontrées, spectres d'hôtes et taux d'infestation. Mémoire de D.E.A. de Botanique, Université de Cocody-Abidjan, $51 \mathrm{p}$.

Traoré D., Da K. P. et D. Soro. 2003. Lutte contre les plantes vasculaires parasites du karité, dans le Nord de la Côte d'Ivoire : Cas du parc naturel à karité de Tengrela. Université de Cocody, U.F.R. Biosciences, Laboratoire de Botanique. Rapport du P.E.P. A.I.S.A.-Cl, $116 \mathrm{p}$. 